\title{
Computers
} \& Structures

PERGAMON

\section{The method of finite spheres with improved numerical integration}

\author{
Suvranu De, Klaus-Jürgen Bathe * \\ Department of Mechanical Engineering, Massachusetts Institute of Technology, Room 3-356, 77 Massachusetts Avenue, Cambridge, \\ MA 02139, USA
}

\begin{abstract}
The method of finite spheres was introduced as a truly meshless technique with the goal of achieving computational efficiency in a mesh-free procedure. In this paper we report several new numerical integration rules that result in a significant reduction in computational cost. (c) 2001 Elsevier Science Ltd. All rights reserved.
\end{abstract}

\section{Introduction}

The conceptual development of a truly meshless scheme, i.e. a computational technique that does not depend on a mesh for interpolation or integration purposes, is rather straightforward. A technique of generating interpolation functions having compact support depending only on a scattered distribution of nodal points on the domain needs to be adopted. This allows the interpolation procedure to be independent of a mesh.

A variety of such meshless interpolation techniques is now available; for example, the moving least squares interpolation technique [1], the weighted least squares technique [2] and methods derived using the partition of unity paradigm $[3,4]$. A weighted residual scheme is used to generate the discretized set of equations. The different meshless techniques found in the literature differ, in essence, in their choice of the interpolation functions, the particular form of the weighted residual scheme employed and the procedure of imposing the boundary conditions.

If a Galerkin formulation is used, an additional hurdle is the development of an integration scheme that is also independent of a mesh. Such integration schemes have been specifically developed in the context of the method of finite spheres [5]. If, on the other hand, a

\footnotetext{
* Corresponding author. Tel.: +1-617-253-6645; fax: +1-617253-2275.

E-mail address: kjb@mit.edu (K.J. Bathe).
}

meshless technique adopts the point collocation procedure as the weighted residual scheme (for example, as in the finite point method [2]), then no such difficulty exists as the governing differential equations are used only at the nodal points. But the quality of solution is rather sensitive to the "proper" choice of the collocation points.

The Galerkin formulation is robust and given a reasonably large finite dimensional approximation space and a sufficiently high order integration rule, convergence is assured. Hence the real challenge is to develop a truly meshless scheme that is computationally efficient. The current trend in research in meshless techniques is towards application of the new techniques to diverse problem areas in engineering and not on the very important issue of computational efficiency. However, for any meshless technique to find eventually wide application, it must be reasonably efficient compared to the now classical finite element/finite volume techniques and it should, of course, be reliable. With these considerations in mind, we proposed a truly meshless technique-the method of finite spheres [5].

In the method of finite spheres the discretization is performed using functions that are compactly supported on general $n$-dimensional spheres and the Galerkin weak form of the governing partial differential equations is integrated using specialized numerical integration rules. The relative orientation and the region of overlap of two spheres are completely determined by the coordinates of their centers and their radii and this makes spherical supports attractive. The interpolation functions are 
generated using the partition of unity paradigm [3]. The Shepard functions [6] using cubic spline weighting functions provide a low-cost partition of unity.

In the Galerkin formulation, one of the major computational costs is the numerical integration of the terms in the weak form. In the traditional finite element methods numerical integration is performed most efficiently using Gauss-Legendre product rules on integration domains that are $n$-dimensional cubes or tetrahedra. The Gauss-Legendre quadrature rule ensures arbitrary polynomial accuracy and therefore the stiffness terms (for undistorted elements) are exactly integrated with low cost [7]. In the method of finite spheres, however, the interpolation functions are rational (non-polynomial) functions on domains and effective numerical integration rules have to be developed.

In Ref. [8] we presented a set of integration rules for disks, sectors and "lens" shaped overlap regions of disks and concluded, from actual comparisons of computational costs and some rough theoretical estimates, that the method of finite spheres was about an order of magnitude slower than the traditional finite element technique for two-dimensional problems in linear elastostatics. In this paper we present a set of improved rules on the same computational subdomains and report a significant reduction in computational costs.

The organization of this paper is as follows. In Section 2 we review the interpolation scheme used in the method of finite spheres. In Section 3 we summarize the displacement-based method of finite spheres as well as a displacement/pressure mixed formulation that we developed to overcome volumetric "locking" [9]. In Section 4 we introduce several new integration rules (that, of course, are applicable to both the displacement based method and the displacement/pressure mixed formulation) and present an estimate of how the improved integration rules result in lower computational costs.

\section{Method of finite spheres shape functions}

In this section we briefly recapitulate how we generate low-cost approximation functions using the partition of unity paradigm [3] based on the Shepard partition of unity functions [6].

Let $\Omega \in R^{d}$ ( $d=1,2$ or 3 ) be an open bounded domain and let $\Gamma$ be its boundary (see Fig. 1). Let a family of open spheres $\left\{B\left(\mathbf{x}_{I}, r_{I}\right) ; I=1,2, \ldots, N\right\}$ form a covering for $\Omega$, i.e. $\Omega \subset \bigcup_{I=1}^{N} B\left(\mathbf{x}_{I}, r_{I}\right)$, where $\mathbf{x}_{I}$ and $r_{I}$ refer to the center and radius of the sphere $I$ respectively. We associate a "node" with the geometric center $\mathbf{x}_{I}$ of each sphere. By $S\left(\mathbf{x}_{I}, r_{I}\right)$ we denote the surface of sphere $I$. The spheres may be entirely within the domain (interior spheres) or may have nonzero intercepts with the boundary (boundary spheres), see Fig. 1.

We define a radial weighting function $W_{I}(\mathbf{x})$, of the form $W_{I}(\mathbf{x})=W\left(s_{I}\right)$, where $s_{I}=\left\|\mathbf{x}-\mathbf{x}_{I}\right\|_{0} / r_{I}$, compactly supported on the sphere centered at node $I$ such that

1. $W_{I}(\mathbf{x}) \in C_{0}^{s}\left(B\left(\mathbf{x}_{I}, r_{I}\right)\right), \quad s \geqslant 0$

2. $W_{I}(\mathbf{x}) \geqslant 0 \quad \forall \mathbf{x} \in \Omega$.

We have chosen a cubic spline weighting function of the following form (refer to Ref. [9] for an explanation):

$$
W\left(s_{I}\right)= \begin{cases}\frac{2}{3}-4 s_{I}^{2}+4 s_{I}^{3}, & 0 \leqslant s_{I} \leqslant \frac{1}{2} \\ \frac{4}{3}-4 s_{I}+4 s_{I}^{2}-\frac{4}{3} s_{I}^{3}, & \frac{1}{2} \leqslant s_{I} \leqslant 1 \\ 0, & s_{I}>1 .\end{cases}
$$

The weighting functions define the Shepard partition of unity functions [6]

$\varphi_{I}^{0}(\mathbf{x})=\frac{W_{I}}{\sum_{J=1}^{N} W_{J}}, \quad I=1,2, \ldots, N$

satisfying

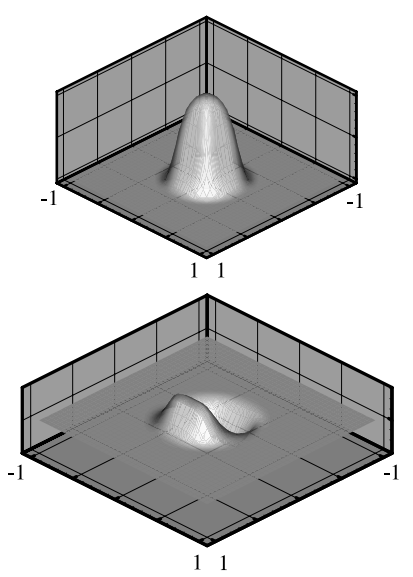

(b)

Fig. 1. (a) A schematic of the method of finite spheres and (b) some shape functions in two dimensions. 
1. $\sum_{I=1}^{N} \varphi_{I}^{0}(\mathbf{x})=\mathbf{1} \quad \forall \mathbf{x} \in \Omega$

2. $\varphi_{I}^{0}(\mathbf{x}) \in C_{0}^{s}\left(R^{d}\right), \quad s \geqslant 0$.

The functions $\left\{\varphi_{I}^{0}(\mathbf{x})\right\}$ satisfy zeroth order consistency, i.e. they ensure that rigid body modes are exactly represented.

To generate approximation spaces with higher order consistency, a local approximation space $V_{I}^{h}=$ $\operatorname{span}_{m \in \mathscr{I}}\left\{p_{m}(\mathbf{x})\right\}$ is defined at each node $I$, where $p_{m}(\mathbf{x})$ is a polynomial or other function and $\mathscr{I}$ is an index set. The superscript $h$ is a measure of the size of the spheres.

The global approximation space $V_{h}$ is generated by multiplying the partition of unity function at each node $I$ with the functions from the local basis

$V_{h}=\sum_{I=1}^{N} \varphi_{I}^{0} V_{I}^{h}$

Hence, any function $v_{h} \in V_{h}$ can now be written as

$v_{h}(\mathbf{x})=\sum_{I=1}^{N} \sum_{m \in \mathscr{I}} h_{I m}(\mathbf{x}) \alpha_{I m}$

where

$h_{I m}(\mathbf{x})=\varphi_{I}^{0}(\mathbf{x}) p_{m}(\mathbf{x})$

and $h_{I m}$ is a basis/shape function associated with the $m$ th degree of freedom $\alpha_{I m}$ of node $I$.

\section{Linear elasticity problems in $R^{2}$}

In this section we apply the method of finite spheres to solve problems in two-dimensional elasticity. Section 3.1 summarizes the pure displacement-based formulation. In Ref. [9] we reported that the pure displacementbased formulation suffers from volumetric "locking" in the case of almost or fully incompressible media. Section 3.2 presents the displacement/pressure mixed formulation [9] to overcome this problem.

\subsection{Displacement-based formulation}

The system of governing differential equations and the boundary conditions for a linear elastic continuum $\Omega \in R^{2}$ with boundary $\Gamma$ can be written as:

$\partial_{\epsilon}^{\mathrm{T}} \tau+\mathbf{f}^{\mathrm{B}}=\mathbf{0} \quad$ in $\Omega$

$\boldsymbol{\epsilon}=\boldsymbol{\partial}_{\epsilon} \mathbf{u}$ in $\Omega$

$\tau=\mathbf{C} \epsilon$ in $\Omega$

$\mathbf{N} \tau=\mathbf{f}^{\mathrm{S}}$ on $\Gamma_{f}$

$\mathbf{u}=\mathbf{u}^{\mathrm{S}}$ on $\Gamma_{u}$.
In Eqs. (4)-(8), $\mathbf{u}, \boldsymbol{\epsilon}$ and $\tau$ are the displacement, strain and stress vectors, $\mathbf{C}$ is the elasticity matrix, $\mathbf{f}^{\mathrm{S}}$ is the prescribed traction vector on the Neumann boundary $\Gamma_{f}, \mathbf{u}^{\mathrm{S}}$ is the vector of prescribed displacements on the Dirichlet boundary $\Gamma_{u}$ (note that the domain boundary $\Gamma=\Gamma_{f} \cup \Gamma_{u}$ ), $\mathbf{f}^{\mathrm{B}}$ is the body force vector (including inertia terms), $\partial_{\epsilon}$ is a linear gradient operator and $\mathbf{N}$ is the matrix of direction cosine components of a unit normal to the domain boundary (positive outwards). In $R^{2}$ these vectors and matrices are written as:

$\mathbf{u}=[u(x, y) v(x, y)]^{\mathrm{T}}$

$\boldsymbol{\epsilon}=\left[\begin{array}{lll}\varepsilon_{x x} & \varepsilon_{y y} & \gamma_{x y}\end{array}\right]^{\mathrm{T}}$

$\tau=\left[\begin{array}{lll}\tau_{x x} & \tau_{y y} & \tau_{x y}\end{array}\right]^{\mathrm{T}}$

$\mathbf{f}^{\mathrm{S}}=\left[f_{x}^{\mathrm{S}}(x, y) f_{y}^{\mathrm{S}}(x, y)\right]^{\mathrm{T}}$

$\mathbf{u}^{\mathrm{S}}=\left[u^{\mathrm{S}}(x, y) v^{\mathrm{S}}(x, y)\right]^{\mathrm{T}}$

$\partial_{\epsilon}=\left[\begin{array}{cc}\partial / \partial x & 0 \\ 0 & \partial / \partial y \\ \partial / \partial y & \partial / \partial x\end{array}\right]$

$\mathbf{N}=\left[\begin{array}{ccc}n_{x} & 0 & n_{y} \\ 0 & n_{y} & n_{x}\end{array}\right]$

$\mathbf{C}=\left[\begin{array}{ccc}c_{11} & c_{12} & 0 \\ c_{12} & c_{11} & 0 \\ 0 & 0 & c_{33}\end{array}\right]$

where for plane stress conditions

$c_{11}=\frac{E}{1-v^{2}}, \quad c_{12}=\frac{E v}{1-v^{2}}, \quad c_{33}=\frac{E}{2(1+v)}$

and for plane strain conditions

$c_{11}=\frac{E(1-v)}{(1+v)(1-2 v)}, \quad c_{12}=\frac{E v}{(1+v)(1-2 v)}$,

$c_{33}=\frac{E}{2(1+v)}$

$E$ and $v$ being Young's modulus and Poisson's ratio of the material, respectively.

\subsubsection{Variational formulation}

We consider the following variational indicator [7]

$\Pi^{*}(\mathbf{u})=\int_{\Omega} \frac{1}{2} \epsilon^{\mathrm{T}}(\mathbf{u}) \mathbf{C} \boldsymbol{\epsilon}(\mathbf{u}) \mathrm{d} \Omega-\mathfrak{R}$.

The term $\mathfrak{R}$ accounts for the externally applied body forces, surface tractions and applied displacements, 
$\mathfrak{R}=\int_{\Omega} \mathbf{u}^{\mathrm{T}} \mathbf{f}^{\mathrm{B}} \mathrm{d} \Omega+\int_{\Gamma_{f}} \mathbf{u}^{\mathrm{T}} \mathbf{f}^{\mathrm{S}} \mathrm{d} \Gamma+\int_{\Gamma_{u}} \mathbf{f}^{u \mathrm{~T}}\left(\mathbf{u}-\mathbf{u}^{\mathrm{S}}\right) \mathrm{d} \Gamma$

where $\mathbf{f}^{u}$ is the traction vector on the Dirichlet boundary and may be expressed as

$\mathbf{f}^{u}=\mathbf{N C} \epsilon(\mathbf{u})$.

Invoking the stationarity of $\Pi^{*}$ we obtain the following weak form:

Find $\mathbf{u} \in H^{1}(\Omega)$ such that

$$
\begin{gathered}
\int_{\Omega} \boldsymbol{\epsilon}^{\mathrm{T}}(\mathbf{v}) \mathbf{C} \boldsymbol{\epsilon}(\mathbf{u}) \mathrm{d} \Omega-\int_{\Gamma_{u}}\left[\boldsymbol{\epsilon}^{\mathrm{T}}(\mathbf{v}) \mathbf{C} \mathbf{N}^{\mathrm{T}} \mathbf{u}+\mathbf{v}^{\mathrm{T}} \mathbf{N} \mathbf{C} \boldsymbol{\epsilon}(\mathbf{u})\right] \mathrm{d} \Gamma \\
=\int_{\Omega} \mathbf{v}^{\mathrm{T}} \mathbf{f}^{\mathrm{B}} \mathrm{d} \Omega+\int_{\Gamma_{f}} \mathbf{v}^{\mathrm{T}} \mathbf{f}^{\mathrm{S}} \mathrm{d} \Gamma-\int_{\Gamma_{u}} \boldsymbol{\epsilon}^{\mathrm{T}}(\mathbf{v}) \mathbf{C} \mathbf{N}^{\mathrm{T}} \mathbf{u}^{\mathrm{S}} \mathrm{d} \Gamma \\
\forall \mathbf{v} \in H^{1}(\Omega)
\end{gathered}
$$

where $H^{1}(\Omega)$ is the first order Hilbert space [7].

\subsubsection{Nodal interpolations}

We have the following approximation for the displacement field

$\mathbf{u}(x, y)=\sum_{J=1}^{N} \sum_{n \in \mathscr{I}} \mathbf{H}_{J_{n}}(x, y) \boldsymbol{\alpha}_{J_{n}}=\mathbf{H}(x, y) \mathbf{U}$

where

$\mathbf{U}=\left[\begin{array}{llllll}\boldsymbol{\alpha}_{10} & \boldsymbol{\alpha}_{11} & \boldsymbol{\alpha}_{12} & \cdots & \boldsymbol{\alpha}_{J n} & \cdots\end{array}\right]^{\mathrm{T}}$

is the vector of nodal unknowns, and

$\boldsymbol{\alpha}_{J_{n}}=\left[\begin{array}{ll}u^{J_{n}} & v^{J_{n}}\end{array}\right]$

is the vector of nodal unknowns at node $J$ corresponding to the $n$th degree of freedom $\left(u^{J n}\right.$ and $v^{J n}$ are the nodal variables for the $x$ - and $y$-direction displacements at node $J$ corresponding to the $n$th degree of freedom). The nodal shape function matrix corresponding to the $n$th degree of freedom is

$\mathbf{H}_{J n}(x, y)=\left[\begin{array}{cc}h_{J n}(x, y) & 0 \\ 0 & h_{J n}(x, y)\end{array}\right]$.

Hence, the discretized versions of Eqs. (10) and (11) are

$\boldsymbol{\epsilon}(x, y)=\sum_{J=1}^{N} \sum_{n \in \mathscr{I}} \mathbf{B}_{J n}(x, y) \boldsymbol{\alpha}_{J_{n}}=\mathbf{B}(x, y) \mathbf{U}$

and

$\boldsymbol{\tau}(x, y)=\sum_{J=1}^{N} \sum_{n \in \mathscr{I}} \mathbf{C B}_{J_{n}}(x, y) \boldsymbol{\alpha}_{J_{n}}=\mathbf{C B}(x, y) \mathbf{U}$

where the strain-displacement matrix $\mathbf{B}(x, y)$ in Eq. (20) is partitioned as

$$
\mathbf{B}(x, y)=\left[\begin{array}{lllll}
\mathbf{B}_{10}(x, y) & \mathbf{B}_{11}(x, y) & \cdots & \mathbf{B}_{J n}(x, y) & \cdots
\end{array}\right]
$$

with

$\mathbf{B}_{J n}(x, y)=\boldsymbol{\partial}_{\epsilon} \mathbf{H}_{J n}(x, y)=\left[\begin{array}{cc}\partial h_{J n} / \partial x & 0 \\ 0 & \partial h_{J n} / \partial y \\ \partial h_{J n} / \partial y & \partial h_{J n} / \partial x\end{array}\right]$.

\subsubsection{Discrete equations}

Using Eqs. (18)-(21) in Eq. (17) we obtain the discretized system of algebraic equations corresponding to node $I$ and degree of freedom $m$

$\sum_{J=1}^{N} \sum_{n \in \mathscr{I}} \mathbf{K}_{I m J n} \boldsymbol{\alpha}_{J n}=\mathbf{f}_{I m}+\hat{\mathbf{f}}_{I m}$.

In this equation the various matrices and vectors are as follows:

$\mathbf{K}_{I m J n}=\int_{\Omega_{I}} \mathbf{B}_{I m}^{\mathrm{T}} \mathbf{C} \mathbf{B}_{J n} \mathrm{~d} \Omega$

$\mathbf{f}_{I m}=\int_{\Omega_{I}} \mathbf{H}_{I m} \mathbf{f}^{\mathrm{B}} \mathrm{d} \Omega$

where $\Omega_{I}=\Omega \cap B\left(\mathbf{x}_{I}, r_{I}\right)$.

If $I$ is a node associated with an "internal sphere", then

$\hat{\mathbf{f}}_{I m}=\mathbf{0}$

from compact support.

If the sphere corresponding to node $I$ has nonzero intercept on the Neumann boundary, then

$\hat{\mathbf{f}}_{I m}=\int_{\Gamma_{f_{I}}} \mathbf{H}_{I m} \mathbf{f}^{\mathrm{S}} \mathrm{d} \Gamma$

where $\Gamma_{f}=\cup_{I \in \mathcal{N}_{f}} \Gamma_{f_{I}}, \mathscr{N}_{f}$ being the index set of such nodes.

On the other hand, if the sphere corresponding to node $I$ has nonzero intercept on the Dirichlet boundary, then

$\hat{\mathbf{f}}_{I m}=\sum_{J=1}^{N} \sum_{n \in \mathscr{I}} \mathbf{K} \mathbf{U}_{I m J_{n}} \boldsymbol{\alpha}_{J n}-\mathbf{f} \mathbf{U}_{I m}$

where

$\mathbf{K U}_{I m J n}=\int_{\Gamma_{u_{I}}} \mathbf{H}_{I m} \mathbf{N C B} \mathbf{B}_{J n} \mathrm{~d} \Gamma+\int_{\Gamma_{u_{I}}} \mathbf{B}_{I m}^{\mathrm{T}} \mathbf{C N}^{\mathrm{T}} \mathbf{H}_{J n} \mathrm{~d} \Gamma$

and

$\mathbf{f} \mathbf{U}_{I m}=\int_{\Gamma_{u_{I}}} \mathbf{B}_{I m}^{\mathrm{T}} \mathbf{C N}^{\mathrm{T}} \mathbf{u}^{\mathrm{S}} \mathrm{d} \Gamma$

where $\Gamma_{u}=\cup_{I \in \mathscr{N}_{u}} \Gamma_{u_{I}}, \mathscr{N}_{u}$ being the index set of such nodes. Note that the stiffness matrix KU is symmetric.

A point to note is that we may incorporate the Dirichlet conditions by the special arrangement of nodes on the boundary as discussed in Ref. [5]. 


\subsection{Displacement/pressure mixed formulation}

For an almost incompressible medium in plane strain conditions, we write the constitutive relationship in the following form (holding, of course, for fully compressible and fully incompressible media as well):

$\tau=-p \mathbf{I}+2 G \boldsymbol{\epsilon}^{\mathrm{D}}$

where $\mathbf{I}$ is the vector corresponding to the Kronecker delta, $G$ is the shear modulus

$G=\frac{E}{2(1+v)}$

and $E$ and $v$ are Young's modulus and Poisson's ratio of the material, respectively; $\epsilon^{\mathrm{D}}$ is the vector of deviatoric strain components,

$\boldsymbol{\epsilon}^{\mathrm{D}}=\boldsymbol{\epsilon}-\frac{\epsilon_{\mathrm{V}}}{3} \mathbf{I}$

where $\epsilon_{\mathrm{V}}$ is the volumetric strain,

$\epsilon_{\mathrm{V}}=\left(\varepsilon_{x x}+\varepsilon_{y y}\right)$.

Note that in Eq. (30) the out-of-plane stress and strain components are now included [7]. The pressure in the body is

$p=-\kappa \epsilon_{\mathrm{V}}$

where the bulk modulus $\kappa$ is

$\kappa=\frac{E}{3(1-2 v)}$.

In addition, we note that the vector of deviatoric stresses

$\tau^{\mathrm{D}}=\tau+p \mathbf{I}$

is related to the vector of deviatoric strains by the following constitutive relationship

$\tau^{\mathrm{D}}=\mathbf{C}^{\mathrm{D}} \boldsymbol{\epsilon}^{\mathrm{D}}$.

\subsubsection{Variational formulation}

We consider the following variational indicator [7]:

$\Pi^{*}(\mathbf{u}, p)=\int_{\Omega}\left[\frac{1}{2} \epsilon^{\mathrm{D}^{\mathrm{T}}}(\mathbf{u}) \mathbf{C}^{\mathrm{D}} \boldsymbol{\epsilon}^{\mathrm{D}}(\mathbf{u})-\frac{1}{2} \frac{p^{2}}{\kappa}-p \epsilon_{\mathrm{V}}(\mathbf{u})\right] \mathrm{d} \Omega-\mathfrak{R}$

The term $\mathfrak{R}$ accounts for the externally applied body forces, surface tractions and applied displacements,

$\mathfrak{R}=\int_{\Omega} \mathbf{u}^{\mathrm{T}} \mathbf{f}^{\mathrm{B}} \mathrm{d} \Omega+\int_{\Gamma_{f}} \mathbf{u}^{\mathrm{T}} \mathbf{f}^{\mathrm{S}} \mathrm{d} \Gamma+\int_{\Gamma_{u}} \mathbf{f}^{u \mathrm{~T}}\left(\mathbf{u}-\mathbf{u}^{\mathrm{S}}\right) \mathrm{d} \Gamma$

where $\mathbf{f}^{u}$ is the traction vector on the Dirichlet boundary $\Gamma_{u}$ and may be expressed as
$\mathbf{f}^{u}=\mathbf{N C}^{\mathrm{D}} \boldsymbol{\epsilon}^{\mathrm{D}}(\mathbf{u})-p \mathbf{N I}$

with $\mathbf{N}$ now containing a fourth column of zeros.

Invoking the stationarity of $\Pi^{*}$ we obtain the following weak form:

Find $\mathbf{u} \in H^{1}(\Omega)$ and $p \in L^{2}(\Omega)$ such that

$$
\begin{gathered}
\int_{\Omega}\left[\boldsymbol{\epsilon}^{\mathrm{D}^{\mathrm{T}}}(\mathbf{v}) \mathbf{C}^{\mathrm{D}} \boldsymbol{\epsilon}^{\mathrm{D}}(\mathbf{u})-\epsilon_{\mathrm{V}}(\mathbf{v}) p\right] \mathrm{d} \Omega-\int_{\Gamma_{u}}\left[\epsilon^{\mathrm{D}^{\mathrm{T}}}(\mathbf{v}) \mathbf{C}^{\mathrm{D}} \mathbf{N}^{\mathrm{T}} \mathbf{u}\right. \\
\left.+\mathbf{v}^{\mathrm{T}} \mathbf{N} \mathbf{C}^{\mathrm{D}} \boldsymbol{\epsilon}^{\mathrm{D}}(\mathbf{u})\right] \mathrm{d} \Gamma+\int_{\Gamma_{u}} \mathbf{v}^{\mathrm{T}} \mathbf{N} \mathbf{I} p \mathrm{~d} \Gamma \\
=\int_{\Omega} \mathbf{v}^{\mathrm{T}} \mathbf{f}^{\mathrm{B}} \mathrm{d} \Omega+\int_{\Gamma_{f}} \mathbf{v}^{\mathrm{T}} \mathbf{f}^{\mathrm{S}} \mathrm{d} \Gamma-\int_{\Gamma_{u}} \boldsymbol{\epsilon}^{\mathrm{D}^{\mathrm{T}}}(\mathbf{v}) \mathbf{C}^{\mathrm{D}} \mathbf{N}^{\mathrm{T}} \mathbf{u}^{\mathrm{S}} \mathrm{d} \Gamma \\
\forall \mathbf{v} \in H^{1}(\Omega) \\
-\int_{\Omega} q\left[\epsilon_{\mathrm{V}}(\mathbf{u})+\frac{p}{\kappa}\right] \mathrm{d} \Omega+\int_{\Gamma_{u}} q \mathbf{I}^{\mathrm{T}} \mathbf{N}^{\mathrm{T}} \mathbf{u} \mathrm{d} \Gamma \\
=\int_{\Gamma_{u}} q \mathbf{I}^{\mathrm{T}} \mathbf{N}^{\mathrm{T}} \mathbf{u}^{\mathrm{S}} \mathrm{d} \Gamma \quad \forall q \in L^{2}(\Omega)
\end{gathered}
$$

where $H^{1}(\Omega)$ and $L^{2}(\Omega)$ are the first order Hilbert space and Lebesgue space of square integrable functions, respectively.

\subsubsection{Nodal interpolations}

We use the same approximation for the displacement field as in Eq. (18), and choose the following approximation for the pressure field

$p(x, y)=\sum_{J=1}^{N} \sum_{n \in \mathscr{I}} h_{J n}^{p}(x, y) p_{J n}=\mathbf{H}_{p}(x, y) \mathbf{P}$

where $\mathbf{P}=\left[\begin{array}{llllll}p_{10} & p_{11} & p_{12} & \cdots & p_{J n} & \cdots\end{array}\right]^{\mathrm{T}}$ is the vector of nodal point unknowns corresponding to the pressure degrees of freedom. The shape function $h_{J n}^{p}(x, y)$ at node $J$ corresponding to the $n$th degree of freedom is also generated using the partition of unity paradigm. This construction of the pressure approximation space results in a continuous pressure field.

It should be noted, however, that the choice of the displacement and pressure interpolation spaces is critical to the success of the mixed formulation scheme. In Ref. [9] we have identified several combinations of these two spaces that result in a stable formulation.

The approximations for the strains in Eqs. (32) and (33) are

$\boldsymbol{\epsilon}^{\mathrm{D}}(x, y)=\sum_{J=1}^{N} \sum_{n \in \mathscr{I}} \mathbf{B}_{J n}^{\mathrm{D}}(x, y) \boldsymbol{\alpha}_{J n}=\mathbf{B}^{\mathrm{D}}(x, y) \mathbf{U}$

and

$\epsilon_{\mathrm{V}}(x, y)=\mathbf{B}_{\mathrm{V}}(x, y) \mathbf{U}$

where $\mathbf{B}^{\mathrm{D}}$ and $\mathbf{B}_{\mathrm{V}}$ are the corresponding strain interpolation matrices. 


\subsubsection{Discrete equations}

Using the displacement and pressure approximations in Eqs. (41) and (42) we obtain the following discrete sets of equations corresponding to node $I$ and degree of freedom $m$

$\sum_{J=1}^{N} \sum_{n \in \mathscr{I}}\left[\begin{array}{ll}\mathbf{K}_{u u_{I m / n}} & \mathbf{K}_{u p_{I m / n}} \\ \mathbf{K}_{u p_{I m / n}}^{\mathrm{T}} & \mathbf{K}_{p p_{I m J n}}\end{array}\right]\left\{\begin{array}{c}\boldsymbol{\alpha}_{J n} \\ p_{J n}\end{array}\right\}=\left\{\begin{array}{c}\mathbf{f}_{I m} \\ \mathbf{0}\end{array}\right\}+\widehat{\mathbf{f}}_{I m}$

where

$$
\begin{aligned}
\mathbf{K}_{u u_{I m J n}} & =\int_{\Omega_{I}} \mathbf{B}_{I m}^{\mathrm{DT}} \mathbf{C}^{\mathrm{D}} \mathbf{B}_{J n}^{\mathrm{D}} \mathrm{d} \Omega \\
\mathbf{K}_{u p_{I m J n}} & =-\int_{\Omega_{I}} \mathbf{B}_{V m}^{\mathrm{T}} h_{J n}^{p} \mathrm{~d} \Omega \\
\mathbf{K}_{p p_{I m J n}} & =-\frac{1}{\kappa} \int_{\Omega_{I}} h_{I m}^{p} h_{J n}^{p} \mathrm{~d} \Omega
\end{aligned}
$$

and

$\mathbf{f}_{l m}=\int_{\Omega_{I}} \mathbf{H}_{l m} \mathbf{f}^{\mathrm{B}} \mathrm{d} \Omega$

where $\Omega_{I}=\Omega \cap B\left(\mathbf{x}_{I}, r_{I}\right)$. If $I$ is a node associated with an "interior sphere", then, of course

$\hat{\mathbf{f}}_{I m}=\mathbf{0}$

If the sphere corresponding to node $I$ has a nonzero intercept on the Neumann boundary $\Gamma_{f}$, then

$\hat{\mathbf{f}}_{I m}=\left\{\begin{array}{c}\int_{\Gamma_{f_{I}}} \mathbf{H}_{I m} \mathbf{f}^{\mathrm{S}} \mathrm{d} \Gamma \\ \mathbf{0}\end{array}\right\}$

where $\Gamma_{f}=\cup_{I \in \mathcal{N}_{f}} \Gamma_{f_{I}} ; \mathscr{N}_{f}$ being the index set of such nodes.

On the other hand, if the sphere corresponding to node $I$ has a nonzero intercept on the Dirichlet boundary $\Gamma_{u}$, then

$\hat{\mathbf{f}}_{I m}=\sum_{J=1}^{N} \sum_{n \in \mathscr{F}}\left[\begin{array}{cc}\mathbf{K} \mathbf{U}_{u u_{I m J n}} & \mathbf{K} \mathbf{U}_{u p_{I m J n}} \\ \mathbf{K} \mathbf{U}_{u p_{I m J n}}^{\mathrm{T}} & \mathbf{0}\end{array}\right]\left\{\begin{array}{c}\boldsymbol{\alpha}_{J n} \\ p_{J n}\end{array}\right\}-\left\{\begin{array}{l}\mathbf{f U}_{u_{I m}} \\ \mathbf{f U}_{p_{I m}}\end{array}\right\}$

where

$\mathbf{K} \mathbf{U}_{u u_{I m J n}}=\int_{\Gamma_{u_{I}}} \mathbf{H}_{I m} \mathbf{N} \mathbf{C}^{\mathrm{D}} \mathbf{B}_{J n}^{\mathrm{D}} \mathrm{d} \Gamma+\int_{\Gamma_{u_{I}}} \mathbf{B}_{I m}^{\mathrm{DT}} \mathbf{C}^{\mathrm{D}} \mathbf{N}^{\mathrm{T}} \mathbf{H}_{J n} \mathrm{~d} \Gamma$

$\mathbf{K U}_{u p l m / n}=-\int_{\Gamma_{u_{I}}} \mathbf{H}_{I m} \mathbf{N} \mathbf{I} h_{J n}^{p} \mathrm{~d} \Gamma$ $\mathbf{f} \mathbf{U}_{u_{I m}}=\int_{\Gamma_{u_{I}}} \mathbf{B}_{I m}^{\mathrm{DT}} \mathbf{C}^{\mathrm{D}} \mathbf{N}^{\mathrm{T}} \mathbf{u}^{\mathrm{S}} \mathrm{d} \Gamma$

and

$\mathbf{f U}_{p_{I m}}=-\int_{\Gamma_{u_{I}}} h_{I m}^{p} \mathbf{I}^{\mathrm{T}} \mathbf{N}^{\mathrm{T}} \mathbf{u}^{\mathrm{S}} \mathrm{d} \Gamma$

where $\Gamma_{u}=\cup_{I \in \mathscr{N}_{u}} \Gamma_{u_{I}} ; \mathscr{N}_{u}$ being the index set of such nodes.

\section{Improved numerical integration schemes}

Efficient numerical integration of the terms in the local weak forms without using a background mesh is challenging because the shape functions are rational (nonpolynomial) functions and the integration domains are spheres or truncated spheres. Moreover, the overlaps of spheres give rise to general "lens" shaped regions. Hence, specialized integration rules are needed. In Ref. [5] we concentrated on two-dimensional domains and developed Gaussian product rules that have certain degrees of polynomial accuracy. We reported that about 144 integration points were required per disk.

In this section we discuss several improved numerical integration rules for two-dimensional conditions. The integration domains that we consider are "interior disks" (disks that have nonzero intercepts with the domain boundary), "boundary sectors" (disks intercepted by the domain boundary) and the "lens" shaped regions of overlap of two disks.

\subsection{Integration on an interior disk}

Let us consider the solution of Poisson's equation on a square domain as shown in Fig. 2(a) using a regular arrangement of nine nodes and a linear local basis. A typical term of the stiffness matrix is

$K_{I m J n}=\int_{\Omega_{I}}\left(\frac{\partial h_{I m}}{\partial x} \frac{\partial h_{J n}}{\partial x}+\frac{\partial h_{I m}}{\partial y} \frac{\partial h_{J n}}{\partial y}\right) \mathrm{d} \Omega$

where $I, J \in\{1, \ldots, 9\}$ and $m, n \in\{0,1\} . h_{I m}$ is the shape function at node $I$ corresponding to the $m$ th degree of freedom. In Fig. 2 we show some of the integrands corresponding to the node at the center of the square $(I=5)$. We observe that it is quite difficult to obtain global polynomial fits to these functions. Hence a high order Gauss integration rule was required in Ref. [5].

It is possible to develop piecewise quadrature formulas by subdividing the disk into subdomains and developing simple quadrature rules on these subdomains. Such a scheme is expected to perform better than the Gaussian product rules since the integration is then performed in a piecewise manner. 

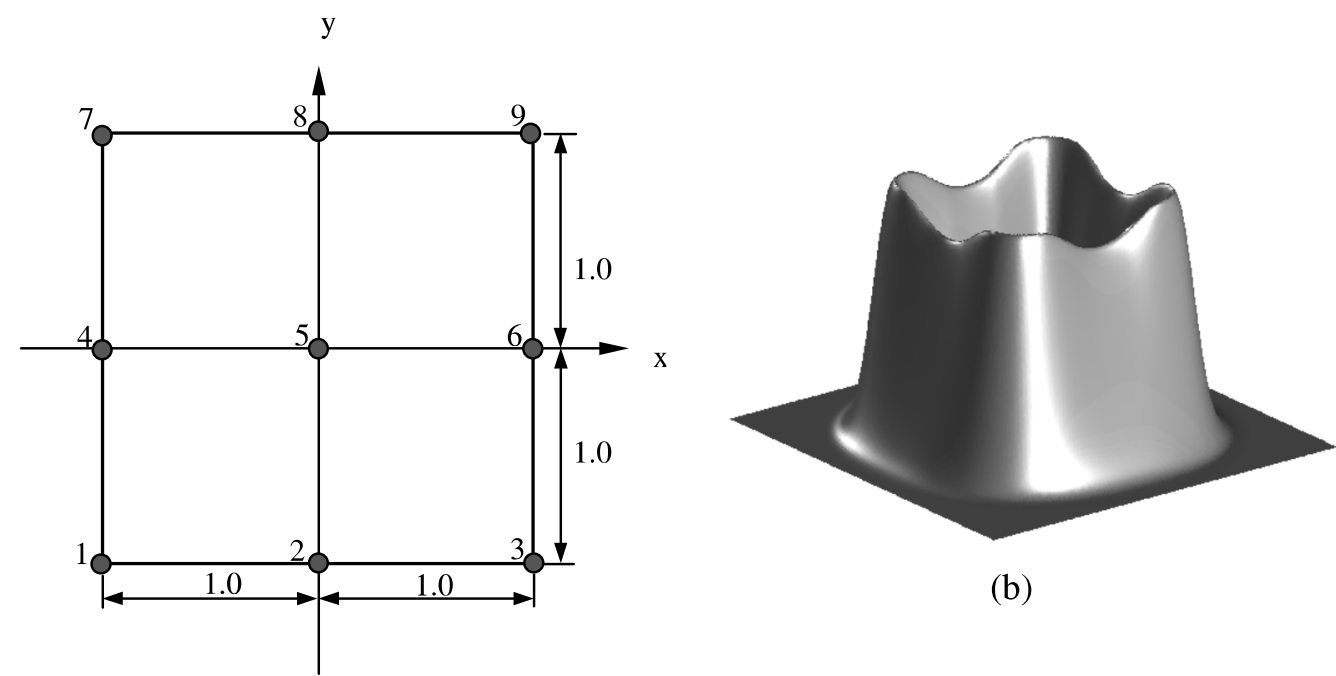

(b)

(a)

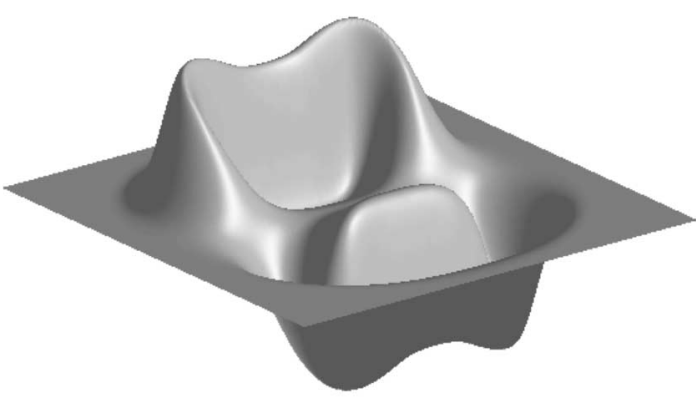

(c)

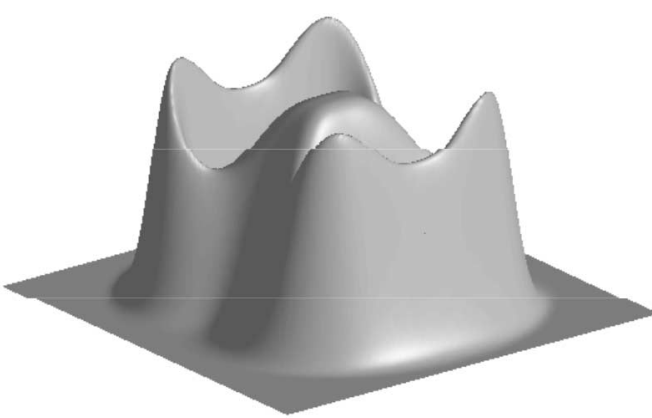

(d)

Fig. 2. (a) A regular arrangement of nine nodes is shown on the domain on which a Poisson problem is defined. Some of the integrands in Eq. (57), namely (b) $K_{5050}$, (c) $K_{5051}$ and (d) $K_{5151}$ are shown corresponding to the node $I=5$.

We have implemented a piecewise midpoint quadrature rule for the interior disk (see Fig. 3(a)) by subdividing the disk using concentric circles and radial lines and evaluating the integral on each of the subdomains as the area of the subdomain multiplied by the integrand evaluated at the centroid of the subdomain. Hence, for the integral of a function $f(x, y)$ on a disk $(\Omega)$ of radius $R_{0}$ we use the following approximation

$\iint_{\Omega} f(x, y) \mathrm{d} x \mathrm{~d} y \simeq \sum_{i=1}^{n_{\theta}} \sum_{j=1}^{n_{r}} D_{i j} f\left(r_{j} \cos \theta_{i}, r_{j} \sin \theta_{i}\right)$

where $n_{\theta}$ is the number of sectors in which the disk is subdivided and $n_{r}$ is the number of subdivisions along each radius. Here

$r_{j}=\frac{j^{2}-j+1 / 3}{j-1 / 2} \Delta r$

$\theta_{i}=(i-1 / 2) \Delta \theta$ where

$\Delta r=\frac{R_{0}}{n_{r}}$

$\Delta \theta=\frac{2 \pi}{n_{\theta}}$.

The weight

$D_{i j}=\left(j-\frac{1}{2}\right) \Delta \theta(\Delta r)^{2}$

is the area of the subdomain and is independent of the circumferential direction.

In Ref. [5] we used, for this integral, the following integration rule due to Peirce [10] which provides an arbitrary polynomial accuracy of degree $k=4 m+3$, $m=0,1,2, \ldots$, in $x=r \cos \theta$ and $y=r \sin \theta$ 


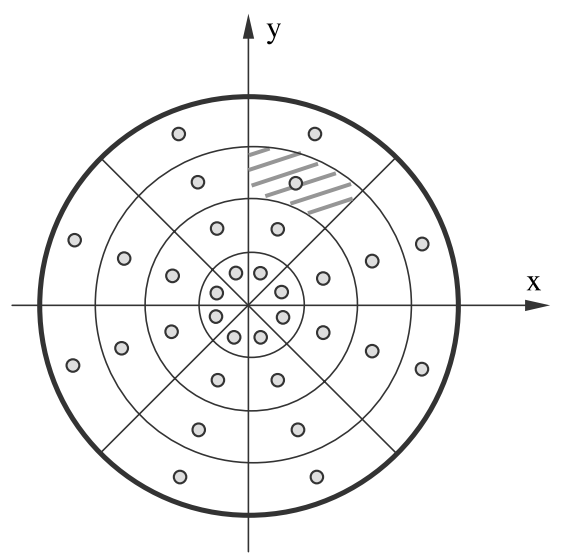

(a)

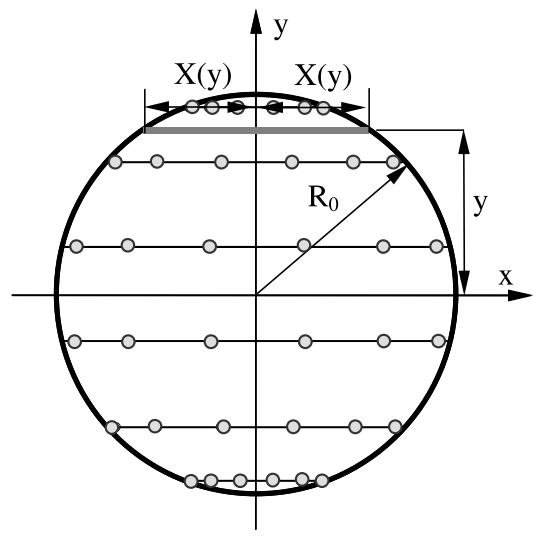

(c)

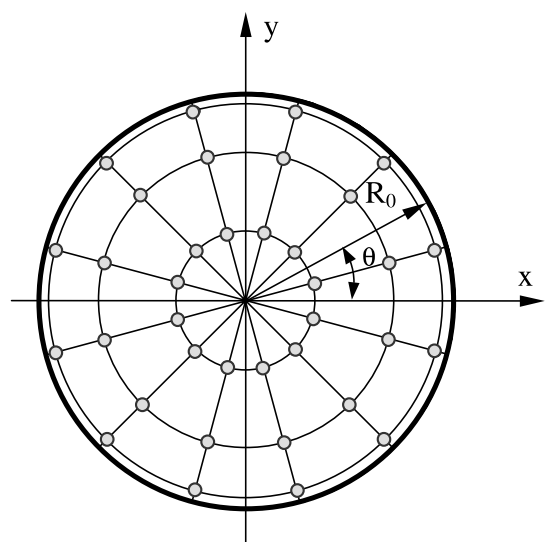

(b)

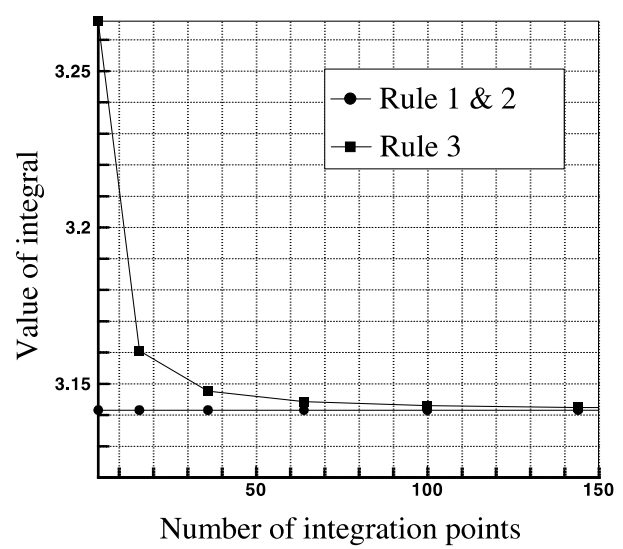

(d)

Fig. 3. Integration on an "interior" disk of radius 1.0. In (a) the integration points corresponding to the piecewise midpoint quadrature rule (Rule 1) are shown. In this rule the integration points are the centroids of the subdomains (hatched) while the areas are the integration weights. In (b) the integration points corresponding to the integration rule in Eq. (59) are shown (Rule 2). In (c) the integration stations corresponding to a Gauss-Legendre product rule (Rule 3) are shown. In (d) Rules 1, 2 and 3 are used to evaluate the area of the disk.

$\iint_{\Omega} f(x, y) \mathrm{d} x \mathrm{~d} y \simeq \sum_{i=1}^{4(m+1)} \sum_{j=1}^{m+1} D_{i j} f\left(r_{j} \cos \theta_{i}, r_{j} \sin \theta_{i}\right)$

where

1. $\theta_{i+1}-\theta_{i}=\frac{2 \pi}{k+1}, \quad i=1,2, \ldots, 4(m+1)$

2. $D_{i j}=\frac{\pi}{4(m+1) P_{m+1}^{\prime}\left(r_{j}^{2}\right)} \int_{0}^{R_{0}^{2}} \frac{P_{m+1}\left(r^{2}\right)}{r^{2}-r_{j}^{2}} \mathrm{~d} r^{2}, \quad j=1,2, \ldots, m+1$

3. The $r_{i}^{2}$ are the $m+1$ zeros of $P_{m+1}\left(r^{2}\right)$, the Legendre polynomial in $r^{2}$ of degree $m+1$, orthogonalized on $\left[0, R_{0}^{2}\right]$.

Since a Gauss-Chebyshev rule automatically arises in the $\theta$-direction, the integration points are on equally spaced radii and the integration weights are independent of angular position (see Fig. 3(b)).

Both this rule and the piecewise midpoint quadrature rule (58) are superior to a simple Gauss-Legendre product rule on the disk used in [11]

$$
\begin{aligned}
\iint_{\Omega} f(x, y) \mathrm{d} x \mathrm{~d} y & =\int_{y=-R_{0}}^{R_{0}} \int_{x=-X(y)}^{X(y)} f(x, y) \mathrm{d} x \mathrm{~d} y \\
& \simeq \sum_{i=1}^{N_{x}} \sum_{j=1}^{N_{y}} D_{i j} f\left(x_{i}, y_{j}\right)
\end{aligned}
$$

where $N_{x}$ and $N_{y}$ are the number of integration points chosen along the $x$ - and $y$-directions, respectively, and $D_{i j}=W_{i}^{x} W_{j}^{y}$ is the product of the usual Gaussian weights $W_{i}^{x}$ and $W_{j}^{y}$ for the $x$ - and $y$-directions (see Fig. 3(c)). 
This integration rule is inefficient which is demonstrated in Fig. 3(d) where we consider the simple problem of computing the area of a unit circle (where $f(x, y)=1$ ) using these three rules.

In Fig. 4 we compare the convergence for the integral in Eq. (57) for the node $I=5$ for $m=0$ and 1 when the rules (58) and (59) are used. In rule (58) equal numbers of sectors and concentric circles are chosen. It can be seen that the simple mid point quadrature rule (58) offers convergence within 49 integration points on the disk.

\subsection{Integration on a boundary sector}

"Boundary sectors" are the disks that have nonzero intercept on the domain boundary. In Ref. [5] we categorized the boundary sectors into two major groups depending on the angle $\varphi_{0}$ that the radii joining the

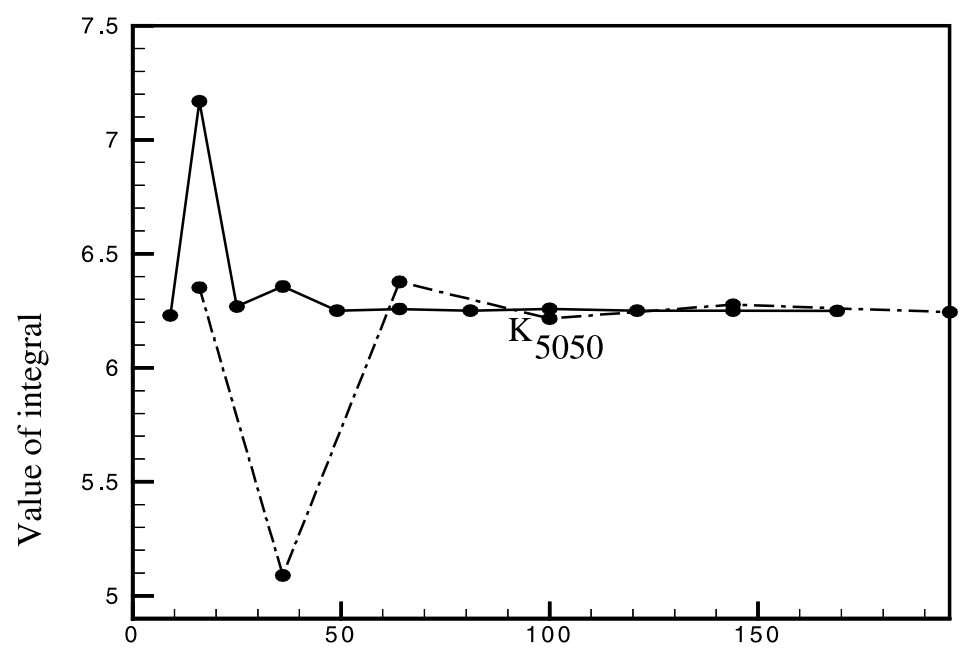

(a)

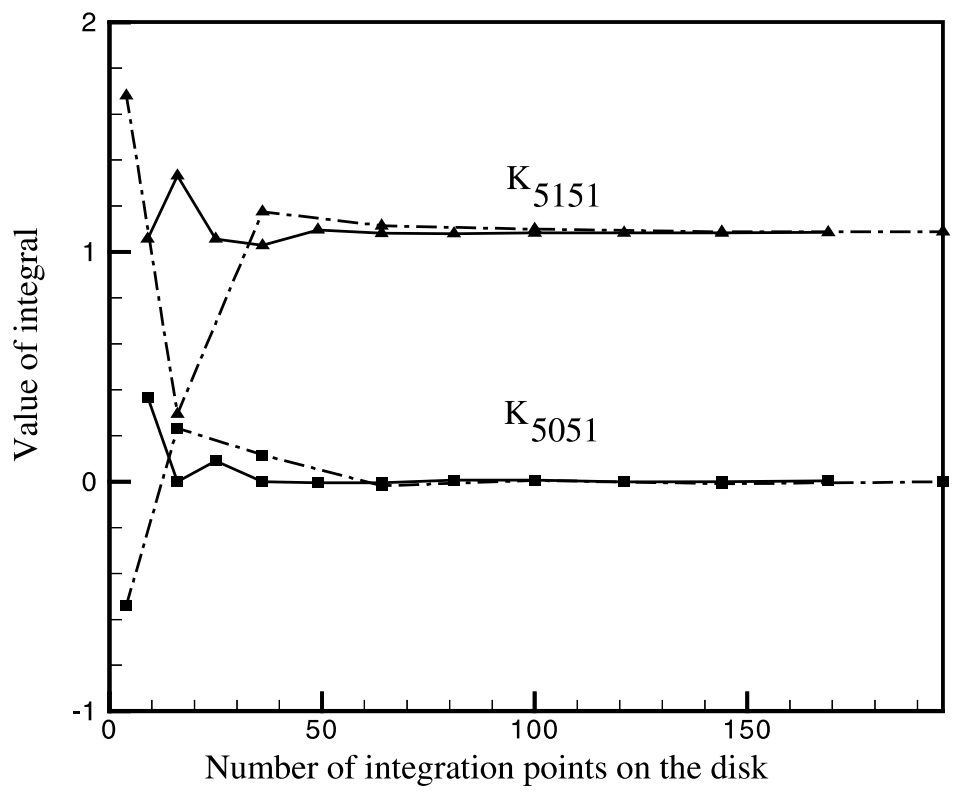

(b)

Fig. 4. Convergence for three integrals in Eq. (57) is shown in (a) and (b), corresponding to $I=5$ and $m=0$ and 1 , using two different integration schemes. The solid lines correspond to the piecewise midpoint quadrature rule and the chain dotted lines correspond to the rule in Eq. (59). Notice that in (a) the integration requires over 100 points to converge (we used 144 points) when using the rule in Eq. (59), whereas 49 points are sufficient when the piecewise midpoint rule is used. 
center of the disk to the two intercepts of the disk on $S$ make interior to the domain:

Type I sector: $\varphi_{0} \leqslant \pi$ (see Fig. 5(a)). The piecewise midpoint quadrature rule (58) applies with the modification

$\Delta \theta=\frac{\varphi_{0}}{n_{\theta}}$
In Ref. [5] we proposed the following "engineering solution",

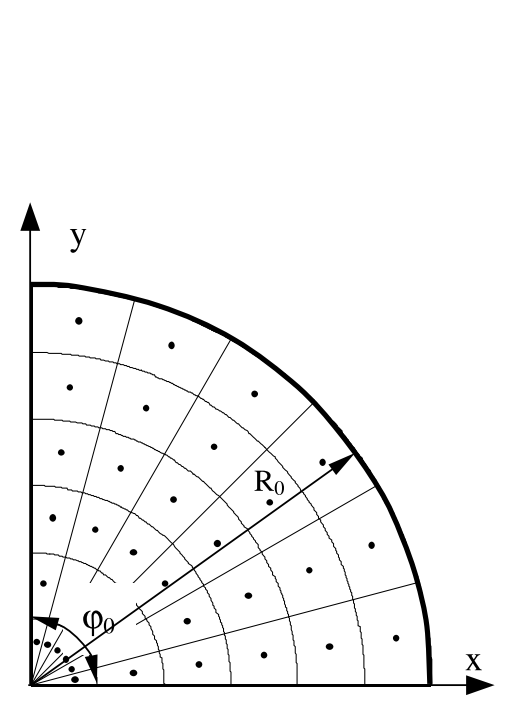

(a)

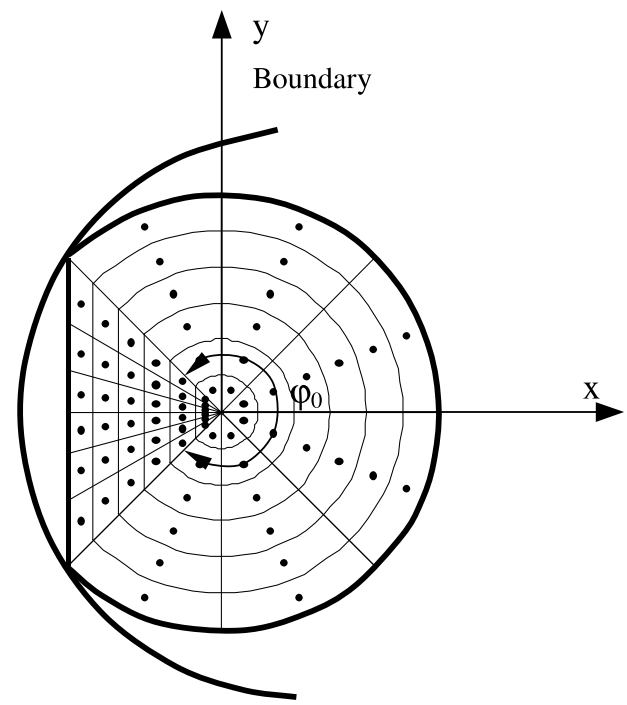

(b)

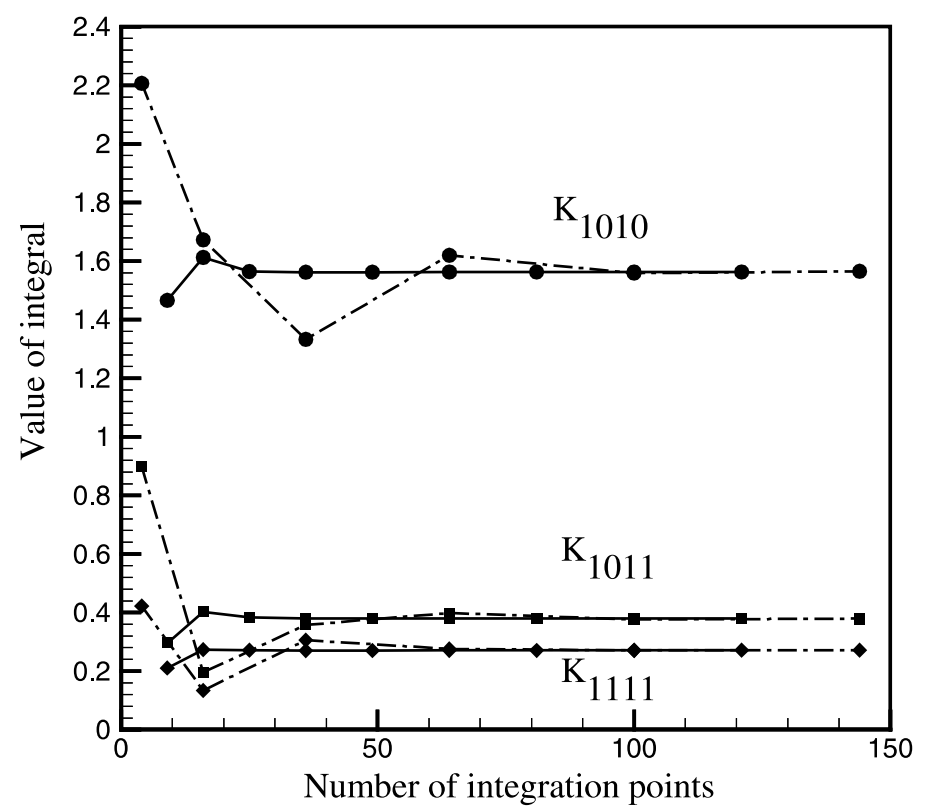

(c)

Fig. 5. Integration points on a boundary sector. (a) Type I boundary sector with $\varphi_{0} \leqslant \pi$. (b) Type II boundary sector with $\varphi_{0}>\pi$. (c) Convergence for three integrals as in Eq. (57) is shown, corresponding to $I=1$ and $m=0$ and 1 , using two different integration schemes. The solid lines correspond to the piecewise midpoint quadrature rule and the chain dotted lines correspond to the "engineering solution" in Eq. (61). Notice the superior convergence properties of the piecewise midpoint rule for these integrals. 
where $D_{i j}=A_{i} B_{j}$ with $A_{i}$ being the usual Gaussian weights on an interval $\left[-\theta_{0}, \theta_{0}\right]$ and

$B_{j}=\frac{1}{2 P_{m+1}^{\prime}\left(r_{j}^{2}\right)} \int_{0}^{R_{0}^{2}} \frac{P_{m+1}\left(r^{2}\right)}{r^{2}-r_{j}^{2}} \mathrm{~d} r^{2} \quad j=1,2, \ldots, m+1$.

The integration points $r_{i}$ are the positive square roots of the zeros of the Legendre polynomial $P_{m+1}\left(r^{2}\right)$ in $r^{2}$ of degree $m+1(m=0,1,2, \ldots)$, orthogonalized on $\left[0, R_{0}^{2}\right]$. However, the radial coordinates of the integration points $\theta_{j}$ are chosen as the zeros of the Legendre polynomial $P_{4(m+1)}(\theta)$ in $\theta$ of degree $4(m+1)$, orthogonalized on $\left[-\theta_{0}, \theta_{0}\right]$.

In Fig. 5(c) we compare the convergence for several integrals of the form (57) where the sector corresponding to node 1 in Fig. 2(a) is considered. Clearly the piecewise midpoint quadrature rule outperforms the previously proposed rule (61).

Type II sector: $\varphi_{0}>\pi$ (see Fig. 5(b)). We may integrate over this type of boundary sector by first decomposing it into a sector for which the rule of the type I sector can be used and a triangle as shown in Fig. 5(b). A piecewise midpoint quadrature rule is used for the triangular region as well.

\subsection{Integration on the lens}

In Ref. [8] we concluded that a Gauss-Legendre product rule of the form

$$
\begin{aligned}
\iint_{\Omega_{I J}} f(x, y) \mathrm{d} x \mathrm{~d} y & =\int_{y=-y_{0}}^{y_{0}} \int_{x=-X_{1}(y)}^{X_{2}(y)} f(x, y) \mathrm{d} x \mathrm{~d} y \\
& \simeq \sum_{i=1}^{N_{x}} \sum_{j=1}^{N_{y}} D_{i j} f\left(x_{i}, y_{j}\right)
\end{aligned}
$$

can be used to numerically evaluate $\iint_{\Omega_{I J}} f(x, y) \mathrm{d} x \mathrm{~d} y$, where $\Omega_{I J}=B\left(\mathbf{x}_{I}, r_{I}\right) \cap B\left(\mathbf{x}_{J}, r_{J}\right) \neq 0$ is the "lens" shaped region of overlap of two disks (see Fig. 6(a)). It was also noted that such a rule requires a rather large number of points to integrate $f(x, y)=1$ on the domain, as shown in Fig. 6(c). However, we noted in Ref. [8] that this rule

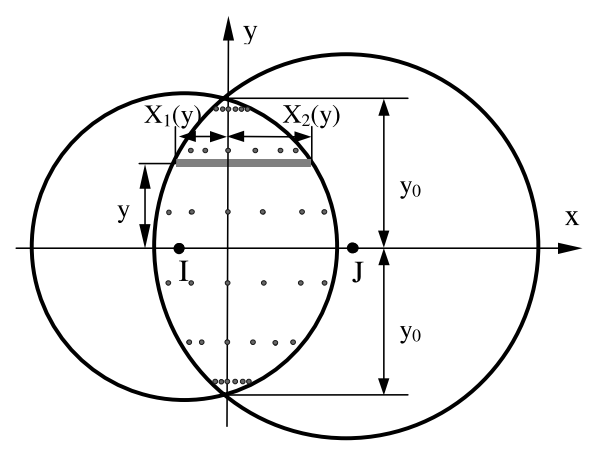

(a)

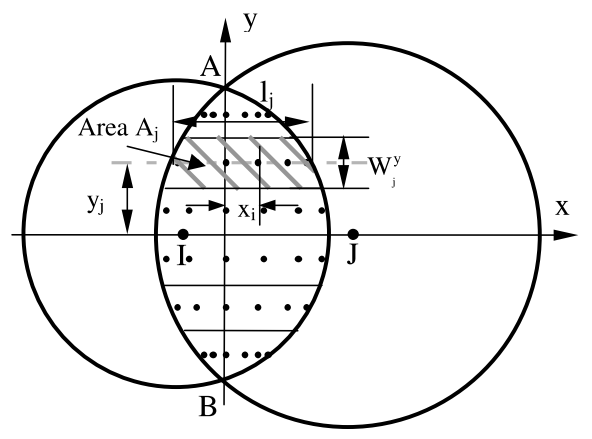

(b)

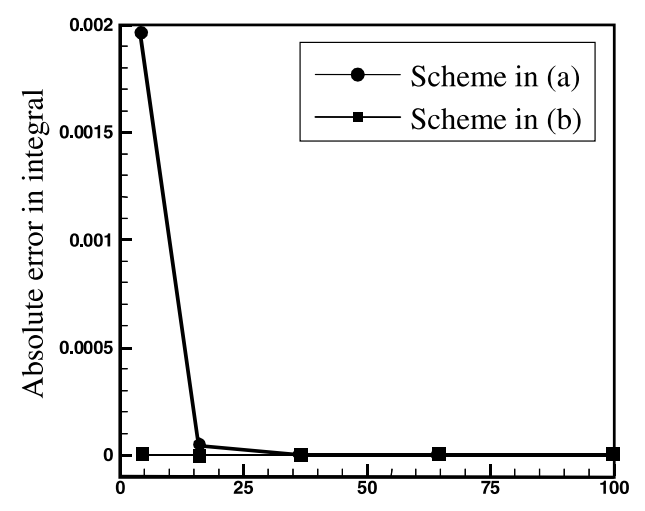

Number of integration points

(c)

Fig. 6. Numerical integration on the lens. Some integration points generated using a Gauss-Legendre product rule, see (a), and piecewise midpoint quadrature rule, see (b), of Section 4.3 are shown on the intersection of two disks of radii 0.8 and 1.0 , respectively, with center-to-center distance of 0.9. Absolute errors as a function of number of integration points when $f(x, y)=1$ is shown in (c). Note that the piecewise midpoint quadrature rule is effective. 
is superior to using a mapping of the lens to a unit circle as proposed in Ref. [11] since the Jacobian required in that case is a complicated function.

In this section we describe a new integration scheme to evaluate the integral on the lens (see Fig. 6(b)). We choose $N_{y}$ integration points along the line $A B$. These points can correspond to either a Gauss quadrature scheme or a piecewise midpoint quadrature scheme. We subdivide the lens into strips of width equal to the integration weights $W_{j}^{y}$. This is possible since $\sum_{j=1}^{N_{y}} W_{j}^{y}=$ length $(A B)$. The area of the $j$ th strip, $A_{j}$, may be computed analytically.

Now we draw a straight line, of length $l_{j}$, parallel to the $x$-axis through each integration point along $A B$ and choose $N_{x}$ integration points along each of these lines. Once again, either a piecewise midpoint or a Gaussian quadrature scheme may be used. Let $W_{i}^{x}$ be the weight associated with the $i$ th integration point, such that $l_{j}=\sum_{i=1}^{N_{x}} W_{i}^{x}$. We may now write down an integration rule

$\iint_{\Omega_{I J}} f(x, y) \mathrm{d} x \mathrm{~d} y \simeq \sum_{i=1}^{N_{x}} \sum_{j=1}^{N_{y}} D_{i j} f\left(x_{i}, y_{j}\right)$

where
$D_{i j}=\frac{A_{j} W_{i}^{x}}{l_{j}}$.

Notice that the integration weight $D_{i j}$ is the fraction of the area of the $j$ th strip and $\sum_{i=1}^{N_{x}} D_{i j}=A_{j}$. If $f(x, y)=1$, then this integration rule is exact (see Fig. 6(c)). Let us denote, by scheme 1 the case when a piecewise midpoint quadrature rule is used along $A B$ as well as along the $x$ axis and by scheme 2 the case when piecewise midpoint rules are used along $A B$ but a Gauss-Legendre rule is used along the $x$-axis.

In Fig. 7 we consider the convergence for three integrals of the type (57). Three different numerical integration schemes are compared: scheme 1 , scheme 2 and our previous integration rule in Eq. (62). We observe that schemes 1 and 2 are comparable and each is better than the rule in Eq. (62). We choose to use scheme 1 in our computations.

\subsection{Computational costs}

The improved integration rules result in a substantial reduction in computational cost for the pure displacement based as well as the displacement/pressure mixed formulation schemes presented in Section 3. In Ref. [8]

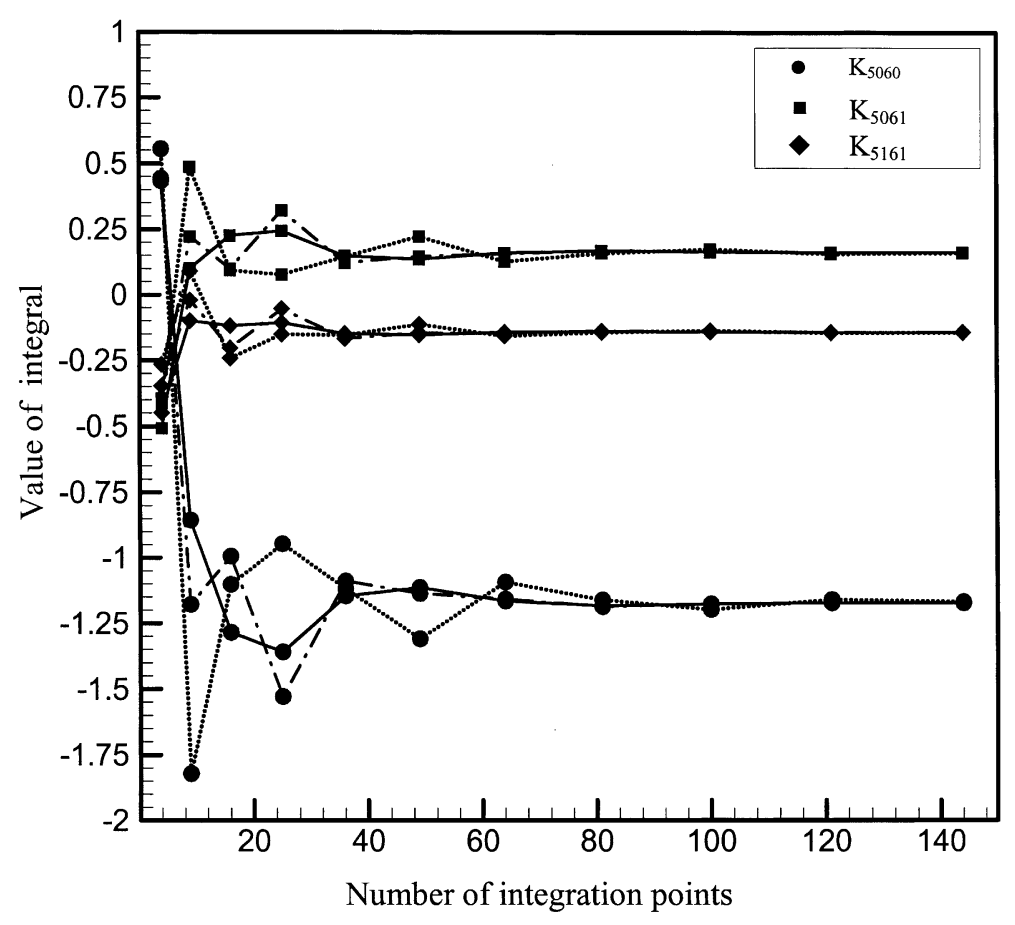

Fig. 7. Convergence for three integrals as in Eq. (57) using the integration rules on the lens. Results shown correspond to the integral on the intersection of disks 5 and 6 using three different integration schemes. The solid lines correspond to a piecewise midpoint quadrature rule along the $x$ - and $y$-directions (scheme 1). The chain dotted lines correspond to a piecewise midpoint quadrature rule along the $y$-direction and a Gauss quadrature rule along the $x$-direction (scheme 2 ) and the dotted lines correspond to a GaussLegendre product rule as in Eq. (62). 
we considered the displacement-based method of finite spheres and estimated the computational cost in solving a general elliptic problem in $d$-dimensions $(d=1,2$ or 3$)$. In addition, we compared this cost with the expense of a similar meshless scheme using the moving least squares approximants as well as the finite element technique. We concluded that the method of finite spheres was computationally much more efficient than the moving least squares based method but was about an order of magnitude slower than the traditional finite element technique for two-dimensional problems in linear elastostatics.

Assuming that the computational cost of a weighting function or its derivative in the method of finite spheres and the cost of computation of a finite element shape function or its derivative are of the same order of magnitude, the ratio of the cost of computation of the global stiffness matrices in the method of finite spheres $\left(T_{K}^{\mathrm{MFS}}\right)$ and the finite element technique $\left(T_{K}^{\mathrm{FEM}}\right)$ may be estimated as (see Ref. [8])

$\frac{T_{K}^{\mathrm{MFS}}}{T_{K}^{\mathrm{FEM}}}=O\left(\frac{n^{2} N_{g}^{\mathrm{MFS}} N^{\mathrm{MFS}}\left(M^{\mathrm{MFS}}\right)^{2}}{N_{g}^{\mathrm{FEM}} N^{\mathrm{FEM}} M^{\mathrm{FEM}}}\right)$

where the $O$ notation signifies asymptotic upper bound to within a constant. The number of nodes is $N$ and each node is assumed to have an average connectivity of $M$, i.e. the support of each node is assumed to have nonzero overlaps with an average of $(M-1)$ other nodal supports (superscripts MFS and FEM refer to the method of finite spheres and the finite element method, respectively). The number of functions used in the local basis of each node in the method of finite spheres is denoted by $n . N_{g}^{\mathrm{MFS}}$ is the number of integration points per sphere and $N_{g}^{\mathrm{FEM}}$ is the number of Gaussian integration points per finite element.

Let us consider the example of the square cantilevered plate in plane strain with uniformly distributed loading on the top surface that we used in Ref. [8]. We have rerun the problem using the improved numerical integration schemes that we have described in this paper. We have observed that a $8 \times 8$ regular nodal arrangement (with quadratic consistency) provides a solution (in strain energy) which is comparable in accuracy with the solution provided by a $25 \times 25$ mesh of nine-noded finite elements. We estimate

$N^{\mathrm{FEM}}=2601 ; \quad N^{\mathrm{MFS}}=64 ;$

$N_{g}^{\mathrm{FEM}}=9 ; \quad N_{g}^{\mathrm{MFS}}=49 ;$

$M^{\mathrm{FEM}} \sim 25 ; \quad M^{\mathrm{MFS}} \sim 4 ;$

$n=6$

and obtain
$\frac{T_{K}^{\mathrm{MFS}}}{T_{K}^{\mathrm{FEM}}} \sim 3$.

This result is quite close to the comparison of actual computational times. In Ref. [8] we noticed that this ratio was 9 . The reduction is due to the fact that we now use 49 integration points per node as opposed to 144 used before.

If we assume that the total computational cost can be broken down into the cost of computation of the global stiffness matrix and the solution of the set of algebraic equations, a consequence of the estimate presented in the last paragraph is that in terms of total computational cost the method of finite spheres is now about two to five times slower than the finite element technique for linear elastostatic problems in two dimensions.

\section{Concluding remarks}

Computational efficiency is the key to the eventual success of a meshless technique. The choice of the computational subdomains, the functions used for interpolation, the techniques applied to impose the Dirichlet boundary conditions and perform numerical integration need to be considered in detail in the development of a computationally efficient scheme.

In the method of finite spheres we have chosen lowcost approximation functions and effective techniques for applying the Dirichlet boundary conditions. The emphasis of our current research is on the development of computationally efficient integration rules. The cost of computation of the global stiffness matrix scales linearly with the number of integration points used per sphere. Hence an integration scheme that is three times more efficient than our previous one implies that we have reduced the computational cost substantially.

The developments reported in this paper suggest that it is possible to obtain a meshless technique which is efficient when compared with classical discretization methods. Of course, more efficient integration schemes should still be sought, and the development of efficient techniques of distributing the nodal points, selecting the radii of the spheres for acceptable accuracy, and computing the intersections of spheres with general boundaries need still to be addressed.

\section{References}

[1] Nayroles B, Touzot G, Villon P. Generalizing the FEM: diffuse approximation and diffuse elements. Computat Mech 1992;10:307-18.

[2] Oñate E, Idelsohn S, Zienkiewicz OC, Taylor RL. A finite point method in computational mechanics. Applications to convective transport and fluid flow. Int J Num Meth Eng 1996;39:3839-66. 
[3] Melenk JM, Babuška I. The partition of unity finite element method: basic theory and applications. Comput Meth Appl Mech Eng 1996;139:289-314.

[4] Duarte CA, Oden JT. H- $p$ clouds - an $h-p$ meshless method. Num Meth Partial Differential Equations 1996;12:673-705.

[5] De S, Bathe KJ. The method of finite spheres. Computat Mech 2000;25:329-45.

[6] Shepard D. A two-dimensional interpolation function for irregularly spaced data. Proc 23rd Nat Conf ACM, 1968. p. 517-24.

[7] Bathe KJ. Finite element procedures. Englewood Cliffs, NJ: Prentice Hall; 1996.
[8] De S, Bathe KJ. Towards an efficient meshless computational technique: the method of finite spheres. Eng Computat 2001;18:170-92.

[9] De S, Bathe KJ. Displacement/pressure mixed interpolation in the method of finite spheres. Int J Num Meth Eng 2001;51:275-92.

[10] Peirce WH. Numerical integration over the planar annulus. J Soc Ind Appl Math 1957;5(2):66-73.

[11] Atluri SN, Kim HG, Cho JY. A critical assessment of the meshless local Petrov-Galerkin (MLPG) and local boundary integral equation (LBIE) methods. Computat Mech 1999;24:348-72. 